\title{
«Ehrfurcht vor dem Leben» als Leitbild für eine zeitgemässe Ethik*
}

Andreas Steiner

\footnotetext{
Dieser Artikel fasst die wichtigsten Gedanken zusammen, die in folgendem Buch ausführlich dargelegt und diskutiert werden: Steiner A. Das Leben, unser höchstes Gut. Albert Schweitzers Ethik im 21. Jahrhundert. Freiburg i. Br.: Herder; 2006.
}

Korrespondenz:

Dr. med. et lic. phil. Andreas Steiner Hanfroosenweg 13 CH-8615 Wermatswil

Tel. 0449945049

ansteiner@ntx.ch
Vor rund zehn Jahren, noch bevor ich mich entschlossen hatte, Philosophie zu studieren, nahm ich an einem an der Universität Zürich von einem Mediziner gehaltenen öffentlichen Vortrag über Sinn und Ziel von Genmanipulationen bei Tieren teil. Als anschliessend dem Publikum die Möglichkeit geboten wurde, sich zu äussern, erlaubte ich mir, folgende Frage zu stellen: «Nach welchem philosophischen Prinzip lassen sich Eingriffe in das Erbgut von Lebewesen ethisch rechtfertigen?» Der Dozent gab diese Frage an den Ethiker weiter, der neben ihm sass. Dieser antwortete: «Heute wird die ethische Grundlage jeweils den äusseren Gegebenheiten angepasst, d.h., sie wechselt, je nachdem, was gerade die Fragestellung ist.»

Ich muss zugeben, dass mich diese Antwort schockiert hatte und dass mir die Anmassung bzw. die Hybris, die dahintersteckt, bis heute keine Ruhe lässt. Ich, der ich damals heimkehrte, nachdem ich während rund zwanzig Jahren als Arzt in Afrika und Südamerika gearbeitet hatte, musste bald erfahren, dass bei uns wichtige Probleme in Naturwissenschaft, Medizin, Politik, Wirtschaft und im Sozialen, die eine ethische Stellungnahme verlangen, öfters so lange analysiert und zerredet werden, bis sie sich vom moralischen Standpunkt aus im Sinne derjenigen, deren handfeste Interessen sich hinter solchen Problemen verstecken, rechtfertigen lassen. Mit anderen Worten: Rational-ethisches Debattieren läuft oft auf ein Zurechtbiegen hinaus, um dem, was den Menschen und der Umwelt aufgedrängt werden soll, ein Schutzmäntelchen überzuziehen, damit es sich als moralisch sauber vertreten lasse.

Wie viele andere Menschen bin ich jedoch davon überzeugt, dass wir einen festen Anhaltspunkt brauchen, auf den wir ethische Fragen rückbeziehen können, ein ruhendes, unverrückbares Prinzip, aus dem heraus wir unser Handeln analysieren, beurteilen und ethisch rechtfertigen können. Lange Zeit hatte die Religion einen solchen Anhaltspunkt dargestellt, für viele Menschen sind jedoch heute Rechtfertigungen über die Religion obsolet geworden.

Dennoch führt das Suchen nach einer Grundlegung für die Ethik immer auch zu religiösen Fragen. Wenn die Grundlegung Allgemeingültigkeit beanspruchen soll, hat sie etwas mit Tran- szendenz zu tun. Auf empirischem Wege jedenfalls kann die Grundlegung für die Ethik nicht erfolgen, da aufgrund blosser Beobachtung und Erfahrung kein Prinzip gefunden werden kann, von dem sich umfassende ethische Grundsätze herleiten lassen. Allenfalls können alltagspraktische Regeln gefunden werden, die klug sein mögen und sich auf gewisse Situationen anwenden lassen. Solche Regeln werden beispielsweise von den Ethikkommissionen aufgestellt, Allgemeingültigkeit können sie jedoch nicht beanspruchen. Um zu einer allgemeingültigen ethischen Grundlegung zu gelangen, muss zunächst intuitiv verfahren werden. Das intuitiv Gefundene muss sich dann deduktiv als Prinzip ausweisen lassen, und zwar so, dass trotz seiner postulierten Allgemeingültigkeit ein Bezug zu Tatsachen und bestehenden Verhältnissen einsehbar und durchführbar ist. Das Herausarbeiten der ethischen Grundlegung erfordert ein Vorgehen, das spekulatives Denken und Praxis gleichermassen berücksichtigt. Jürgen Habermas drückt sich ähnlich aus, wenn er schreibt: «[...] nur über Ideen kann eine Lebensordnung legitime Geltung erwerben» [1].

Für Immanuel Kant sind Ideen keine sinnlich erfahrbaren Wirklichkeiten, er spricht ihnen jedoch in praktischer Hinsicht eine wichtige regulative Funktion zu.

Als Grundlage unserer Ethik brauchen wir so glaube ich - eine allgemeinverbindliche Idee, die uns vorausschwebt wie einst der Stern den Drei Königen aus dem Morgenland, eine Idee, die uns auf dem Weg des Guten leuchtet, die uns Kraft verleiht und die unsere Hoffnung auf Glückseligkeit nicht versiegen lässt. Wir brauchen ein geistiges Leitbild, dem jeder folgen kann, das nie aufhört, uns als eine höhere Bestimmung herauszufordern. Auf die Erde herabgezogen und mittels unserer verfahrenstechnisch ausgerichteten Vernunft in seine Bestandteile zerlegt, würde es aufhören, ein Leitbild für unser Denken und Handeln zu sein.

Ausgehend von dem, was ich als unser «höchstes Gut» bezeichne, habe ich versucht, eine solche Idee, ein solches Leitbild als unverrückbaren Grund für ethische Entscheide, wie sie auch vom Arzt immer wieder verlangt werden, $\mathrm{zu}$ postulieren. 
Als unser «höchstes Gut» hat sich mir das Prinzip des «Lebens in seinem umfassenden Sinn» aufgedrängt. Ich verstehe darunter das Prinzip, das uns alle in unserer Existenz bedingt und in unserer Alltäglichkeit aufrechterhält und das uns alle verbindet, nicht nur mit den anderen Menschen, sondern mit allen Lebewesen, also auch mit Tieren und Pflanzen.

Dass wir als Menschen in die allgemeine Lebenswelt eingegliedert und für unser Dasein auf das Mitdasein der anderen Lebewesen angewiesen sind, dürfte weder natur- noch sozialwissenschaftlich zu bestreiten sein. Auch dass über die Evolution alle Lebewesen miteinander verwandt sind, ist unbestrittene Tatsache. Als Ärzte sind wir darüber besonders gut informiert. Nicht zuletzt aber wird diese Verwandtschaft auch in den Mythen der Völker, in denen sich die Seelenverfassung eines jeweiligen menschlichen Kollektivs bildlich ausdrückt, demonstriert.

Leben im umfassenden Sinn als unser «höchstes Gut» ist eine Idee, und als Idee gehört sie zum Absoluten. Das Absolute des umfassenden Lebens manifestiert sich jedoch konkret und damit sinnlich in jedem Lebewesen, sei dieses nun ein Mensch, ein Tier oder eine Pflanze.

Intuitiv erfahren wir Leben als unsere Seele, als unseren innersten Kern, als unser eigentliches Wesen. Sowohl in der antiken Philosophie wie im Christentum wird dem Leben göttlicher Ursprung zugeschrieben. Leben wird als etwas verstanden, das in die Materie gelegt ist, das uns zukommt als göttlicher Hauch, als Seelenfunken, als Lebensatem, im Lateinischen mit dem Wort «anima» ausgedrückt, das all diese Bedeutungen in sich vereinigt. In der Medizin begegnet uns das Wort «anima» im Begriff der Re-Animation, in der Wieder-Belebung. Dass metaphorisch gesprochen - der formlose Stoff, aus dem wir gemacht sind, «Leben in sich aufnimmt» und zu dem wird, was jeder von uns in seiner Individualität ist, wird keiner, auch nicht der hartgesottenste Positivist, bestreiten wollen. Er wird vielleicht antworten, Leben sei nichts anderes als Materie, die sich in einem kontinuierlichen Strukturierungsprozess befinde. Doch, so erlaube ich mir zu fragen, was liegt diesem Prozess zugrunde?

Die Ergebnisse und Erfolge der naturwissenschaftlichen und medizinischen Forschung sollen nicht bestritten werden. Wir Ärzte wissen, was und wie etwas funktionieren muss, damit wir von «Leben» sprechen können. Auch gibt es heute verschiedene Hypothesen über das, was sich abgespielt haben könnte, als Leben in seiner primitivsten Form zuerst entstand und sich in immer neue und komplexere Formen verzweigte. Bis heute wissen wir jedoch nicht, warum Leben in seiner Urform angefangen und was es veranlasst hat, sich weiterzuentwickeln, bis es schliesslich die «Krone der Schöpfung», wie wir den Menschen gerne bezeichnen, geschaffen hat.

Dem Leben in seiner universalen Bedeutung besondere Aufmerksamkeit entgegenzubringen sollte uns heute, wo wir Menschen mehr denn je zerstörerisch in natürliche Vorgänge eingreifen, ein besonderes Anliegen sein. Im Denken des Arztes sollte «Leben als umfassende Grundlage» und damit als «höchstes Gut» allen Daseins immer präsent sein. Denn in der Wirtschaft und in der Politik herrschen heute nicht Ideen und Projekte, die dem Leben zugewandt sind, als Leitbild vor, sondern die Modellvorstellung des «Homo oeconomicus»:

Der «Homo oeconomicus» ist der Prototyp eines modern-utilitaristisch eingestellten Menschen, der Erfolg nur dann anerkennt, wenn sich dieser in einer möglichst grossen Zahl ausdrükken lässt, und der deshalb alle seine Urteile nach quantitativen Gesichtspunkten fällt. Profitmaximierung um jeden Preis für sich selbst und für sein Unternehmen bestimmt sein Tun, als ob den Menschen nur dadurch Glück zuteil würde. Qualitative Kriterien, wie humanitäre oder soziale Fortschritte, sind für ihn zweitrangig oder belanglos. Werte, die sich nicht in Zahlen ausdrücken lassen, also nicht «be-zahl-bar» sind, fallen kaum ins Gewicht.

Denken, das zerteilt und dann wieder addiert oder subtrahiert, bewegt sich auf der unendlichen Zahlenreihe auf und ab. Die Zahlenreihe an sich ist eine Linie, d.h., sie ist eindimensional, und ebenso bewegen sich die Denkprozesse, die sich auf der Zahlenreihe abspielen und sich auf diese beschränken, in nur einer Dimension. Eindimensionalität in ihrer Ausschliesslichkeit jedoch ist das Defizitäre an der Ratio des «Homo oeconomicus».

Die normale menschliche Vernunft kann ihre Denkkapazität auf mindestens drei Dimensionen ausdehnen und im Geist facettenreiche Bilder des Seienden konzipieren und sich gedanklich vorstellen. Die Eindimensionalität des Denkens ist Zeichen einer Verarmung der ursprünglichen menschlichen Denkfähigkeit.

Die defizitäre, eindimensionale Rationalität bestimmt den «Homo oeconomicus» und damit das Leitbild des heutigen Menschen. Dieses Leitbild ging aus der Wirtschaft hervor und wurde von der Politik übernommen.

Wie soll nun umfassendes Leben, das wir als unser höchstes Gut betrachten, ethisch zur Geltung kommen? Aus «Leben» an und für sich 
ergibt sich noch keine ethische Richtung, resultieren noch keine moralischen Verhaltensregeln. Hier greife ich auf Albert Schweitzers Maxime «Ehrfurcht vor dem Leben» zurück, die genau das ausdrückt, was der modernen Gesellschaft heute not tut und was unser ethisches Leitbild sein sollte.

Der Arzt, Theologe und Philosoph Albert Schweitzer erfuhr diese Maxime wie eine Erleuchtung, als er 1915 mit einem Dampfer auf dem Ogowe zu einer Patientin fuhr, um ihn herum nur Urwald und Wasser. Er schreibt darüber: «Die fundamentale Tatsache des Bewusstseins des Menschen lautet: «Ich bin Leben, das leben will, inmitten von Leben, das leben will.> Der denkend gewordene Mensch erlebt die Nötigung, allem Willen zum Leben die gleiche Ehrfurcht vor dem Leben entgegenzubringen wie dem seinen. Als gut gilt ihm, Leben erhalten, Leben fördern, entwickelbares Leben auf seinen höchsten Wert bringen. Als böse: Leben vernichten, Leben schädigen, entwickelbares Leben niederhalten. Dies ist das denknotwendige, universelle, absolute Grundprinzip des Ethischen» [2].

Albert Schweitzer setzt sich mit dem von ihm postulierten Prinzip «Ehrfurcht vor dem Leben» eingehend in seinem Buch «Kulturphilosophie und Ethik» auseinander. Ich will hier nicht näher auf seine Darstellungen und Aussagen eingehen. Wenn man jedoch den Begriff «Ehrfurcht» in der Literatur sucht, stösst man nicht auf viele Hinweise. Eine bedeutende Stelle befindet sich in Goethes Werk «Wilhelm Meisters Wanderjahre»: «Ungern entschließt sich der Mensch zur Ehrfurcht oder vielmehr entschließt sich nie dazu; es ist ein höherer Sinn, der seiner Natur gegeben werden muß, und der sich nur bei Begünstigten aus sich selbst entwickelt, die man auch deswegen von jeher für Heilige, für Götter gehalten» [3].

Ehrfurcht ist eine Haltung, die erworben und von Jugend auf geübt werden muss. Ehrfurcht ist niemandem in die Wiege gelegt, sie muss anerzogen werden. Damit sich Ehrfurcht bei Jugendlichen einstellt, braucht es Erzieher, die selbst gelernt haben, Ehrfurcht zu empfinden und mit ihr umzugehen. Aus der Haltung der Ehrfurcht heraus belassen wir allen Wesen und Dingen, mit denen wir in Kontakt treten, ihre Bedeutung und damit auch ihre Würde, gleichzeitig bringen wir ihnen die gebührende Achtung entgegen.

Um Ehrfurcht empfinden zu können, braucht es eine bestimmte Erkenntnis, die man sich aneignen muss. Sie zu vermitteln ist Aufgabe der Eltern und der Bildungsanstalten. Grundlage dieser Erkenntnis ist das Wissen über Bedeutung und Wert der Wesen und der Dinge, mit denen man zu tun hat. Es ist ein Gewahrwerden, das über das alltägliche Wissen hinausgeht. Fachlich geht es darum, einesteils das naturwissenschaftliche, soziale und kulturelle Wissen zu vertiefen, andernteils die Aufmerksamkeit auf Zusammenhänge hinzuführen, die nicht ohne weiteres offen zutage liegen.

Mit «Ehrfurcht vor dem Leben» sind zwei weitere Prinzipien verbunden: erstens liebende Zuwendung zu allem, was lebt, und zweitens uneingeschränkte Verantwortung für das Lebendige.

Beide Prinzipien sind in Albert Schweitzers Werken angetönt. Ich möchte sie aber noch mehr in den Vordergrund rücken, als er es getan hat, und sie als unverrückbaren Bestandteil der «Ehrfurcht vor dem Leben» postulieren, also: «Ehrfurcht vor dem Leben mit liebender Zuwendung zu allem, was lebt, und mit uneingeschränkter Verantwortung».

Erst wenn sich Ehrfurcht vor dem Leben und Liebe gegenseitig ergänzen, ereignet sich ein Vollkommensein zwischen einem Ich und einem Du. Liebe empfinden zu können ist eine Grundbedingung des eigenen Glücksempfindens, das Hand in Hand mit dem Glücksempfinden der anderen geht.

Aus der Zuneigung, die im Gefühl der Liebe gründet, wird schliesslich Zuwendung; man fängt an, sich aktiv um das Gegenüber zu kümmern. Liebe zusammen mit Ehrfurcht vor dem Leben ist eine Gemütshaltung, die sich allem Lebendigen gegenüber öffnet, sich an seinem Gedeihen erfreut und dieses fördert. Gerade hier fühlen wir uns als Ärzte angesprochen. Ich erfuhr das eindrücklich bei meiner ärztlichen Tätigkeit in Afrika, Peru und Südarabien. Im Lauf der Jahre erkannte ich, dass es nur eine Weise gibt, mit den Menschen, die in jenen Gegenden wohnen, erfolgreich zusammenzuarbeiten: nämlich einesteils so, dass ich ihnen mit der ihrem Menschsein gebührenden Ehrfurcht gegenübertrete, über die ich mich ihnen als ebenbürtig verbunden fühle, und andernteils so, dass auf die liebende Zuwendung, die Grundhaltung meiner ärztlichen Tätigkeit sein soll, eine Antwort ihrerseits erfolgen kann. Für eine erfolgreiche Entwicklungszusammenarbeit ist solches Verhalten Voraussetzung.

Was die Verantwortung betrifft, habe ich mich von Hans Jonas' Buch «Das Prinzip Verantwortung» anleiten lassen. Jonas hebt dort hervor, dass sich bei jedem von uns neben der Verantwortung für die uns umgebende heutige Welt, für seine Familie, Freunde usw. auch eine Verantwortung für das herausbilden sollte, was 
in ferner Zukunft sein wird, d.h. für das Weitergehen des Lebens auf der Erde. Denn der Zweck, den wir unserer Existenz als solcher zuschreiben, bleibt für uns nur gewahrt, wenn wir die Gewissheit haben, dass das, was an uns menschlich ist, in zukünftigen Generationen weiterbestehen wird. Unsere Bemühungen, unser Handeln, unsere Ideen wären kaum noch sinnvoll, wenn wir befürchten müssten, dass es in einiger Zeit keine menschlichen Wesen auf der Erde mehr geben würde.

Das Gefühl der Verantwortlichkeit für das Leben wird durch das Gefühl der Ehrfurcht für alles Lebendige in mir geweckt, denn gegenüber dem, was ich ehrfürchtig als mir verbunden erkenne, habe ich ein Interesse, dass es erhalten bleibe. Dieses Interesse ist ein grundsätzliches, denn weil ich mich verbunden fühle, betrifft es auch meine eigene Existenz. Es nötigt mich zur Verantwortung, und ich kann nicht anders, als das Verantwortungsgefühl, das in mir gegenüber dem Leben heranwächst, wahrzunehmen und entsprechend zu handeln.

Ehrfurcht ist eine Haltung, die Kindern und Jugendlichen in der Erziehung vermittelt werden muss. In «Wilhelm Meisters Wanderjahre» wird sie als das wichtigste Ziel aller Erziehungsbemühungen hervorgehoben, also noch wichtiger als der Erwerb theoretischer und praktischer Kompetenzen und wichtiger als jedes Fachwissen.

Aus Ehrfurcht vor dem Leben wird die innere Einstellung der liebenden Zuwendung und der uneingeschränkten Verantwortung hervorgehen. Es entsteht jene Grundlegung der Ethik, aus der heraus man sein Leben gestaltet, handelt, seinen Beruf ausübt und Entscheidungen in Wirtschaft und Politik fällt. Hier können wir als Ärzte, die wir uns in unserem täglichen Tun den Lebensvorgängen und dem Leiden der Menschen widmen, eine Art von Vorbild sein.

In unseren Bildungsanstalten findet heute leider eine Entwicklung statt, die weit entfernt ist von dem, was man als Vermittlung der Ehrfurcht bezeichnen könnte. Unter dem Druck der Wirtschaft wird bei der Ausbildung auf möglichst viel fachliche Kompetenz und frühe Spezialisierung Wert gelegt. Das, was heute ein Jugendlicher lernt, soll sich später «auszahlen» können; die Zehntausende von Franken, die man in eine Ausbildung investiert, sollen eines Tages als sogenannte «harte Fakten», als Profite, zurückkehren.

Dieser Trend hat auch auf Universitäten und andere Hochschulen übergegriffen. Das von den europäischen Bildungspolitikern eingeführte «Bologna-System» stellt nur den äusseren Rah- men dieses Trends dar. Was bis anhin als umfassende Bildung nach dem humanistisch geprägten Ideal bezeichnet wurde, zu dem versteckt auch die Ehrfurcht vor dem Lebendigen gehörte, hat angeblich ausgedient und soll fortan durch eine Ausbildung ersetzt werden, die sich vorwiegend den Bedürfnissen der Wirtschaft anpasst.

Passiert mit unserem Gesundheitswesen nicht etwas Ähnliches? Wirtschaftliche Überlegungen, rein quantitative Parameter, rücken dort mehr und mehr in den Vordergrund. Amteten beispielsweise früher Ärzte als Hauptverantwortliche für die Spitäler, sind es heute Verwalter und Ökonomen, also Leute, die vorwiegend dem Modell des «Homo oeconomicus» und weniger der «Ehrfurcht vor dem Leben» verpflichtet sind.

Charles Kleiber, schweizerischer Staatssekretär für Bildung und Forschung, schreibt (zitiert in: [4]), Wissen sei heute als Quelle des Profits $\mathrm{zu}$ betrachten und entsprechend $\mathrm{zu}$ managen. Wissenschaft solle deshalb, weg von humanistischen Idealen, dem Markt zugeführt werden.

Jugendliche - und dazu gehören mehr und mehr auch jene, die Medizin studieren - sollen heute in erster Linie $\mathrm{zu}$ «nützlichen Gliedern der Produktionsprozesse» ausgebildet werden, damit sie sich später wie Figuren auf den Schachbrettern der nationalen Betriebe und der multinationalen Konzerne hin und her schieben lassen. Den Mut zum Widerstand und die Kraft zur Eigeninitiative werden sie als Erwachsene nur noch mit Mühe aufbringen, wenn sie Freiheit im Denken nie gelernt haben und wenn sich ein dem Lebendigen zugewandtes Interesse in ihnen nie ausgebildet hat. Wenn der Mensch dem Leben gegenüber gleichgültig geworden sei, bestehe keine Hoffnung mehr, dass er das Gute wählen könne, betont Erich Fromm in einem seiner Bücher [5]. Genau diese Gleichgültigkeit gilt es zu bekämpfen.

Für denjenigen, der sich mit offenem, hoffnungsvoll tätigem Geist dem eigenen Selbst und der Umwelt zuwendet, gibt es kein Prinzip, keine Maxime, kein Postulat, das ihn in stärkerem Masse in den Lebensprozess einbindet und damit sein Leben bestimmt als die mit liebender $\mathrm{Zu}$ wendung und Verantwortlichkeit verbundene Ehrfurcht vor dem Leben. Nach diesem Prinzip muss sich unser Tun ausrichten, sofern es als ein ethisches bezeichnet werden will. Durch unseren Beruf sind wir als Ärzte ohnehin dem Leben zugewandt. Ehrfurcht vor dem Leben gehört zu unserem Berufsethos und ist damit Bestandteil unserer Arbeit, die wir nur dann erfolgreich tun können, wenn wir uns in liebender Zuwendung der Kranken und Leidenden annehmen und be- 
reit sind, die volle Verantwortung für unsere Patienten zu tragen.

Was für den Arzt selbstverständlich ist, sollte jedoch für alle Menschen gelten. Es ist eine Frage der inneren Haltung. Es sollen keine Gebote und keine Regeln aufoktroyiert werden. Denn nur in innerer Freiheit kann sich Ehrfurcht vor dem Leben entwickeln. Es geht um eine sanfte Konditionierung unserer Wachsamkeit und inneren Bereitschaft dem Lebendigen gegenüber, an dem wir alle gleichermassen teilhaben. Sie umfasst Menschen, Tiere und Pflanzen. Ehrfurcht vor dem Leben tritt mit keiner anderen Maxime, mit keinem anderen Prinzip, mit keiner Weltanschauung und mit keinem religiösen Bekenntnis in Konkurrenz. Sie schliesst keine einzige Religion aus, sondern ergänzt und bringt vielmehr zum Vorschein, was in den einzelnen Religionen bereits angelegt ist, und fördert damit die Gemeinsamkeit zwischen diesen. «Ehrfurcht vor dem Leben» wendet sich einzig gegen jene Mentalität, die nur ausbeuten, zerstören und rücksichtslos profitieren und die sich um jede Verantwortung für das Ausgebeutete und Zerstörte drücken will.

\section{Literatur}

1 Habermas J. Theorie des kommunikativen Handelns. Band 1. Frankfurt am Main: Suhrkamp; 1995. S. 265 .

2 Schweitzer A. Gesammelte Werke in fünf Bänden. Hg. Rudolf Grabs. Band 5, Kulturphilosophie und Ethik. München: C. H. Beck; 1974. S. 181.

3 von Goethe JW. Gedenkausgabe der Werke, Briefe und Gespräche. Hg. Ernst Beutler. Band 8, Wilhelm Meisters Wanderjahre. Zürich: Artemis; 1949. S. $170 f$.

4 Jost HU. Kalte Revolution an Schweizer Universitäten. Tages-Anzeiger vom 10. Mai 2006, S. 10, Bezug nehmend auf einen Artikel in «Le Temps» vom 11. Oktober 1999.

5 Fromm E. Die Seele des Menschen. Ihre Fähigkeit zum Guten und zum Bösen. München: Deutscher Taschenbuch Verlag; 2000. S. 172. 